\title{
A Metric for Testing Program Verification Systems ${ }^{\star}$
}

\author{
Bernhard Beckert ${ }^{1}$, Thorsten Bormer ${ }^{1}$, and Markus Wagner ${ }^{2}$ \\ 1 Department of Informatics, Karlsruhe Institute of Technology \\ beckert@kit.edu, bormer@kit.edu \\ 2 School of Computer Science, The University of Adelaide \\ markus.wagner@adelaide.edu.au
}

\begin{abstract}
The correctness of program verification systems is of great importance, and it needs to be checked and demonstrated to users and certification agencies. One of the contributing factors to the correctness of the whole verification system is the correctness of the background axiomatization, respectively the correctness of calculus rules. In this paper, we examine how testing verification systems is able to provide evidence for the correctness of the rule base or the axiomatization. For this, we present a new coverage criterion called axiomatization coverage, which allows to judge the quality of existing test suites for verification systems. We evaluate this coverage criterion at two verification tools using the test suites provided by each tool.
\end{abstract}

\section{Introduction}

Motivation. Correctness of program verification systems is imperative if they are to be used in practice. One may employ formal methods to prove a system or its calculus to be correct. But - as for any other type of software system - testing is of great importance.

In this paper, we bring together proofs and tests not as a combination of both used on a program to be validated, but rather to increase software quality by improving conclusiveness of the verification tool itself. Traditional testing techniques alone are insufficient for this purpose - the typical properties and particularities of program verification systems have to be taken into consideration when designing test suites. It is relevant, for example, that verification systems usually do not just consist of an implementation in an imperative programming language but also include axioms and rules written in a declarative language.

The testing process employed must be systematic and the quality of tests suites has to be evaluated. Objective criteria, such as coverage measures, are needed to demonstrate the dependability of verification systems to users and certification agencies.

\footnotetext{
* Work partially funded by the German Federal Ministry of Education and Research (BMBF) in the framework of the Verisoft XT project under grant 01 IS 07008 . The responsibility for this article lies with the authors.
} 
Topic and Structure of this Paper. In this paper, we present a new coverage criterion for testing program verification systems, called axiomatization coverage. We conducted experiments on two verification tools that measure axiomatization coverage of existing test suites, in order to assess the significance of the proposed coverage metric. Our focus is on system tests (as opposed to testing components of the tools); and we test for functional correctness (not usability etc.). Moreover, we only consider tests that can be executed automatically.

The structure of this paper is as follows: First, in Section 2, we clarify what verification systems we consider and discuss their relevant properties. Section 3 is concerned with the test cases we use and the general set-up for testing. In Section 4 we examine the different correctness properties for which we test and explain which kinds of tests relate to what properties. In Section 5, we define a new notion of test coverage for the declarative (axiomatic) part of verification systems. We report on two case studies in Section 6 in which we have evaluated the new test coverage criterion using test suites for two verification systems. Then, in Section 7, we put our work into the context of related work. Lastly, in Section 8 , we draw conclusions and discuss future work.

\section{Target of Evaluation: Program Verification Systems}

Modern Program Verification Tools. Every program verification system has to perform (at least) two rather separate tasks: (a) handling the program-languagespecific and specification-language-specific constructs, and reducing or transforming them to classical logic expressions, (b) theory reasoning and reasoning in classical logics, for handling the resulting expressions and statements over data types. One can either handle these tasks in one monolithic logic/system, or one can use a combination of subsystems.

In this paper, we concentrate on a paradigm of user interaction with the verification tool termed auto-active verification, and which is used by tools such as VCC [8], or Caduceus [1]. In auto-active verification, the requirement specification, together with all relevant information to find a proof (e.g., loop invariants) is given to the verification tool right from the start of the verification processinteraction hereafter is not possible.

All of the tools mentioned above use several subsystems. A more monolithic approach is taken by the Java Card verification tool KeY [5]; in addition, in $\mathrm{KeY}$, user interaction is possible also during the proof construction stage. In many cases however, $\mathrm{KeY}$ can be used in an auto-active manner without relying on user input during proof construction. For the rest of the paper, we restrict all test cases to be provable without interaction. This allows us to treat VCC and $\mathrm{KeY}$ in the same way.

Imperative versus Declarative System Components. Program verification tools have to capture the program language semantics of the programs to be verified. In some tools this information is mostly stored as one huge axiomatization (e.g., as with logical frameworks like Isabelle/HOL) and the implementation part can be kept relatively small. Other tools (e.g., some static checkers) implicitly contain most of the programming language semantics in their implementation. 
To assure the correctness of program verification tools, it is necessary to validate both parts: the implementation, as well as the axiomatization. Only testing the implementation is not sufficient, even if a high code coverage is achieved.

\section{Test Cases for Program Verification Systems}

As said above, the tests we consider in this paper are system tests, i.e., the verification tool is tested as a whole. Though the correctness of a tool, of course, depends on the correctness of its components and it makes sense to also test these components independently, such unit tests cannot replace testing the integrated verification system. Moreover, not all components are easy to test individually. For example, it is possible (and useful) to unit-test an SMT solver that is used by a tool. But the verification condition generator is hard to test separately as it is very difficult to specify its correct behaviour - more difficult than specifying the correct behaviour of the verification system as a whole. Also, we concentrate on functional tests that can be executed automatically, i.e., usability tests and user-interface properties are not considered.

As is typical for verification tools following the auto-active verification paradigm, we assume that a verification problem consists of a program to be verified and a requirement specification that is added in form of annotations to the program. Which annotations are compatible to a program, i.e., which annotation types exist and in which program contexts a particular annotation is allowed, depends on the given annotation language. If $P$ is a program and $A$ is a set of annotations compatible with $P$, then we call the pair $P+A$.

Definition 1 (Annotation satisfaction). We assume that there is a a definition of when a program $P$ satisfies a specification $S P E C$, denoted by $\models P+S P E C$.

Besides the requirement specification, a verification problem usually contains additional auxiliary annotations that help the system in finding a proof. We assume that all other auxiliary input (e.g., loop invariants) are made part of the testing input, such that the test can be executed automatically.

The possible outcomes of running a verification tool on a test case $P+(R E Q \cup$ $A U X)$ (a verification problem consisting of a program $P$ a requirement specification $R E Q$, and auxiliary annotations $A U X)$ are

proved: A proof has been found showing that the program $P$ satisfies $R E Q \cup$ $A U X$.

not provable: There is no proof (either $P$ does not satisfy $R E Q$ or $A U X$ is not sufficient); the system may provide additional information on why no proof exists, e.g., by a counter example or by showing the current proof state.

timeout: No proof could be found given the allotted resource (time and space).

\section{Testing Different Properties}

Testing a verification system can exhibit various kinds of failures related to different correctness properties. A verification system can be unsound, i.e., verify 
an incorrect program to be correct. There can be completeness failures, where the system fails to find a proof for the correctness of a program. There also can be performance failures, where the system's performance is too low either in terms of resource consumption or in terms of the amount and complexity of auxiliary input the user has to provide to be able to prove a program correct. And, of course, the system may crash. While abnormal termination of software is usually a serious concern, a bug that makes the verification system crash is in general less serious than a completeness failure because it cannot be confused with bugs in the program to be verified (the verification target).

How one can test for different kinds of failures, and thus, correctness properties, is discussed in the following subsections. Table 1 summarizes the different test results and the failures they indicate ${ }^{3}$

\subsection{Testing Soundness}

The most important property of verification tools is soundness. This means that whenever the output for a verification problem $P+S P E C$ is "proved", then the program $P$ indeed satisfies the specification $S P E C$.

To reveal a soundness bug, a test case must consist of a program $P$ and a specification $S P E C$ such that $P$ does not satisfy $S P E C$. The correct answer for such a verification problem is "not provable" or "timeout", while "provable" indicates a soundness failure.

Programs that satisfy their specification cannot reveal soundness bugs-at least not directly. The exception are cases where the expected answer is "timeout" but the system answers "proved". Such an anomaly - that needs to be investigated by the developer - can either stem from an unexpected good performance or from a short but incorrect proof (i.e., a soundness problem).

\subsection{Testing Completeness}

As all sound verification systems must be incomplete (Rice's theorem), sound and complete software verification tools cannot exist. Instead the notion of relative completeness is used, i.e., completeness in the sense that the system (respectively its calculus) would be complete if it had an oracle for the validity of formulas about arithmetic [9].

In practice, however, all of today's program verification systems are not even relatively complete. This is not only due to resource limitations. Verification systems presuppose auxiliary annotations or other user input. Auto-active verification tools do not attempt to generate all missing auxiliary annotations. Such an "annotation generator" would give auto-active verification systems the (theoretical) property of being relatively complete but would be useless in practice (although in theory it can be built). Thus, it is neither given nor expected that a program verification system is relatively complete. In practice, completeness of verification tool means that if the program is correct w.r.t. its given requirement

\footnotetext{
${ }^{3}$ The possibility of abnormal termination exists in all cases and is not included in the table.
} 
specification $R E Q$, then some auxiliary specification $A U X$ or other required user input exists allowing to prove this 4 .

Definition 2 (Annotation completeness). A verifying system $S$ is annotation complete if for each program $P$ and specification $R E Q$ with

$$
\models P+R E Q \quad,
$$

there is a set AUX of annotations such that

$$
\vdash_{S} P+(R E Q \cup A U X),
$$

i.e., $S$ finds a proof for $=P+(R E Q \cup A U X)$.

To reveal a completeness problem, a test case must consist of a program $P$ with annotations $R E Q \cup A U X$ such that (a) $P$ satisfies $R E Q \cup A U X$ and (b) the annotations are strong enough to prove this, i.e., the expected output is "proved".

If the observed output is "not provable", then a completeness failure is revealed. In that case, a proof may exist using a different (stronger) set $A U X^{\prime}$ of auxiliary annotations. That is, the system may or may not be annotation complete. The failed test only shows that the expectation that a proof can be found using the annotation set $A U X$ does not hold. The situation is similar with an observed output "timeout". In that case, the system may just be slower as expected or, worse, there may be no proof using $A U X$ or, worst of all, there may be no proof at all for any annotation set $A U X^{\prime}$. For both kinds of incorrect output ("not provable" and "timeout") the developer has to further investigate what kind of failure occurred.

One may consider incompleteness of a verification tool to be harmless in the sense that it is noticeable: the user does not achieve the desired goal of constructing a proof and thus knows that something is wrong. In practice, however, completeness bugs can be very annoying, difficult to detect, and time-consuming. A user may look for errors in the program to be verified or blame the annotation $A U X$ when no proof is found for a correct program and try to improve $A U X$ while in fact nothing is wrong with it. It is therefore very important to systematically test for completeness bugs.

\section{A New Coverage Criterion: Axiomatization Coverage}

Measuring code coverage is an important method in software testing to judge the quality of a test suite. This is also true for testing verification tools. However, code coverage is not an indicator for how well the declarative logical axioms and definitions are tested that define the semantics of programs and specifications and make up an important part of the system.

To solve this problem, we define the notion of axiomatization coverage. It measures to which extent a test suite exercises the axioms used in a verification system. The idea is to compute the percentage of axioms that are actually used in the proofs or proof attempts for the verification problems that make up a 
Table 1: The different test results and the failures they indicate.

\begin{tabular}{cccc} 
& \multicolumn{3}{c}{ Intended } \\
\cline { 2 - 4 } Observed & proved & not provable & timeout \\
\cline { 2 - 4 } proved & - & unsoundness & $\begin{array}{c}\text { unsoundness or } \\
\text { positive performance } \\
\text { anomaly }\end{array}$ \\
\hline \multirow{2}{*}{ not } & incompleteness or & & incompleteness or \\
provable & $\begin{array}{c}\text { performance failure } \\
\text { w.r.t. required } \\
\text { annotations }\end{array}$ & & positive performance \\
& anomaly
\end{tabular}

test suite. We distinguish two versions: (a) the percentage of axioms needed to successfully verify correct programs (completeness coverage), and (b) the percentage of axioms used in failed proof attempts for programs not satisfying their specification (soundness coverage).

An erroneous axiom may lead to unsoundness or incompleteness or both. The latter effect, where something incorrect can be derived and something correct cannot, is actually quite frequent. Because of that, completeness tests allow to find soundness bugs and vice versa. Nevertheless, one should use both kinds of tests (soundness and completeness) and, thus, both kinds of coverage.

\subsection{Completeness Coverage}

For the completeness version of axiomatization coverage, we define an axiom to be needed to verify a program, if it is an element of a minimal axiom subset using which the verification system is able to find a proof. That is, if the axiom is removed from the subset, the verifier is not able anymore to prove the correctness of the program.

Definition 3. A test case $P+(R E Q \cup A U X)$ covers the axioms in a set $T h$ if $T h \vdash P+(R E Q \cup A U X)$ but $T h^{\prime} \forall P+(R E Q \cup A U X)$ for all $T h^{\prime} \subsetneq T h$.

$A$ test case $P+(R E Q \cup A U X)$ strongly covers the axioms in a set $T h$, if $T h \subseteq T h^{\prime}$ for all sets $T h^{\prime}$ covered by $P+(R E Q \cup A U X)$.

Ideally, the coverage definitions would use logical entailment instead of inference. However, as we want to quantify axiom coverage in practice, because of the inherent incompleteness of the verification tools the coverage metric is based on the inference relation.

As a consequence, the axiom coverage of a test suite w.r.t. a system depends on resource constraints (e.g., number of proof steps allowed, timeout or memory 
limitations) and the implementation of the verification system, most notably the proof search strategy. That implies when calculating axiom coverage, to get reproducible results, performance of the computer the verification tool runs on has to be taken into account. In addition, axiom coverage of a test suite has to be recomputed not only when the axiomatization or test suite changes but also whenever parts of the implementation of the verification tool relevant for proof search are modified.

In general, the minimal set of axioms covered by a given verification problem is not unique. So, the question arises of what to do if a test case covers several different axiom sets.

We chose to follow the more conservative approach to consider only one (non-deterministically chosen) axiom set to be covered by any given test case. A pragmatic reason for that choice is that it is very costly to compute all minimal axiom sets covered by a test case. But our conservative choice has another important advantage: If, for example, there is a logically redundant axiom $A^{\prime}$ that is an instance of some other axiom $A$, and there are verification problems that can be solved either using the more general axiom $A$ or the more special $A^{\prime}$, then there are test cases that cover $A$ and $A^{\prime}$ separately but not at the same time. We will count only one of $A$ and $A^{\prime}$ to be covered by such a single test case. Now there are two situations to consider: (1) $A^{\prime}$ is included in the axiom set for good reason as it leads to better performance (shorter proofs) for a certain class of problems. Then there should be a test case in the test suite that can only be proved using $A^{\prime}$. This test should have sufficiently low resource bounds so that it cannot be verified using $A$, and thus demonstrates the usefulness of $A^{\prime}$-and test $A^{\prime}$. And there should be a different test case that does not fall in the special class to which $A^{\prime}$ applies and that can only be solved using $A$. Then, with the two test cases, both $A$ and $A^{\prime}$ are covered. (2) $A^{\prime}$ is really redundant, i.e., it is not possible to construct a test case that can be verified with $A^{\prime}$ but not with $A$. Then $A^{\prime}$ is indeed akin to dead code (which also cannot be covered by test cases) and should be removed.

The way in which we compute the axioms covered by a test case ensures that the non-deterministic choice of the covered axioms is done in a useful way in case there is more than one possibility (see Sect. 6).

\subsection{Soundness Coverage}

For the soundness version of axiomatization coverage, the above definition of needed axioms based on minimal axiom sets is not useful. Instead, an axiom is used in a failed proof attempt, if it occurs in the proof search, i.e., the verification system actively used the axiom for proof construction. What "used" means depends on the particular verification system and its calculus.

\section{Case Studies}

To evaluate the usefulness of our notion of axiomatization coverage, we conducted two case studies. As verification systems to be tested, we chose the VCC 
tool, as well as the KeY system. For both tools, we chose to evaluate the test suite that is part of the corresponding distribution. In addition, for the KeY tool, we examined a third party test suite.

Computing Axiomatization Coverage in Practice We have implemented a framework that allows for the automated execution and evaluation of tests for both tools that computes the completeness version of axiomatization coverage.

To compute an approximation of the taclet coverage for a completeness test case $P+S P E C$, the procedure is as follows: in a first step, $P+S P E C$ is verified with the verification tool using the complete axiom base available. Besides gathering information on resource consumption of this proof attempt (e.g., number of proof steps resp. time needed), information on which axioms are actually used in the proof are recorded as set $T$ (e.g., by leveraging Z3's option to generate unsatisfiability cores in case of $\mathrm{VCC}^{4}$ resp. parsing KeY's explicit proof object).

In a reduction step, we start from the empty set $C$ of covered axioms. For each axiom $t$ in the set of axioms $T$ used in the first proof run, an attempt to prove $P+S P E C$ using axioms $C \cup(T \backslash\{t\})$ is made. If the proof does not succeed, $t$ is added to set $C$. Axiom $t$ is removed from $T$ and the next proof iteration starts until $T=\varnothing$.

In all these subsequent proof runs, resource constraints are set to twice the amount of resources needed for the first proof run recorded initially. This allows for calculating axiom coverage in reasonable time and ensures comparability of coverage measures between computers of different processing power.

The resulting set of axioms $C$ is only an approximation of the coverage of $P+S P E C$. For precise results the above procedure would have to be repeated with $C$ as input as long as the result is different from the input. For practical reasons we compute taclet coverage using only one iteration for the following case studies.

Note that it currently takes several minutes to compute the minimal axiom set for an average test case. This is acceptable if the coverage is not computed too often, but a considerable speed-up should be possible using heuristics for choosing the axioms to remove from the set. Divide and conquer algorithms, e.g., akin to binary search, seem to be suited to reduce computation times at first glance. However, they do not help in practice: as the reduction step does not start from the whole axiomatization but rather from the subset $T$ of taclets actually used in a proof, only relatively few taclets remain that are not covered and can be discarded in the iterative proof runs. For divide and conquer algorithms to be successful, large sets of taclets which could be discarded at once are needed.

\subsection{Testing the Axiomatization of VCC}

The Architecture and Workflow of VCC In the following we give a short overview of the verification workflow and give a description of the architecture of the VCC tool. For a thorough introduction to the VCC methodology, see [8].

\footnotetext{
4 The coverage experiments in this paper have been produced by an older version of our framework without this feature-however, this only impacts performance of the framework.
} 
Table 2: Coverage measures for the first experiment. earlier version of axiomatization

\begin{tabular}{lrrr}
\hline & total & covered & percentage \\
\hline axioms for C language features & 212 & 84 & $40 \%$ \\
axioms for specification language features & 166 & 102 & $61 \%$ \\
all axioms & 378 & 186 & $49 \%$ \\
\hline \hline \multicolumn{4}{c}{ later version of axiomatization } \\
\hline \hline
\end{tabular}

The VCC tool chain allows for modular verification of $\mathrm{C}$ programs using method contracts and invariants over data structures. Method contracts are specified by pre- and postconditions. These contracts and invariants are stored as annotations within the source code in a way that is transparent to the regular $\mathrm{C}$ compiler. The tool chain translates the annotated $\mathrm{C}$ code into first-order logic. Subsequently, the formulas are given to the SMT solver Z3 [10 together with the prelude (the axiomatization) capturing the semantics of C's built-in operators, etc. Z3 checks whether the verification conditions are entailed by the axiomatization. Entailment implies that the original program is correct w.r.t. its specification.

Axiomatization Coverage Results Using our testing framework, we automatically executed the test cases contained in VCC's test suite and measured both the axiomatization and the code coverage achieved with these tests.

First Experiment. First, we used the test suite of VCC version 2.1.20731.0. It consists of 400 test cases, 202 of which are completeness tests. For comparison, we measured axiomatization coverage for two versions of VCC: version 2.1.20731.0, from which the test suite was taken, and version 2.1.20908.0, which is a version about six weeks further into development. The earlier version of the axiomatization contains 378 axioms out of which 186 were covered (49\%). A classification of these axioms and the different degrees of coverage for different types of axioms is shown in Table 2 .

The later version of the axiomatization contains 384 axioms, of which only 139 were covered (36\%), i.e., axiomatization coverage decreased. Investigations revealed that the reason for this decrease is that axioms were modified, e.g., by removing requirements or by adding predicates. Therefore, the old test suite was less adequate to the newer version of VCC.

Second Experiment. In a second experiment, we used VCC version 2.1.30820.1 (one year after the VCC version used in the first experiment), and the accompanying updated test suite. For comparability reasons we used only the part corresponding to the old test suite. This part now contains a total of 698 test cases, 417 of which are completeness tests (the rest are soundness tests and tests checking for parser errors). The axiomatization now consists of 439 axioms. Of 
these 211 were covered by the completeness tests (48\%), i.e., axiomatization coverage has remained stable as compared to the first experiment. For this second experiment, however, we also computed the code coverage for the part of VCC that is related to the semantics of the programming and the specification language, i.e., the verification condition generator. Using the same test suite of 417 completeness tests, the resulting code coverage turned out to be $70 \%$. This is an interesting result as it shows that axiomatization coverage $(48 \%)$ can be quite a bit lower than code coverage $(70 \%)$. And it is evidence that axiomatization coverage is independent of code coverage. Therefore, axiomatization coverage should indeed be considered in addition to code coverage to judge the quality of a test suite.

Other Insights. Additional investigations showed a further difference between code coverage and axiomatization coverage: The code coverage of individual test cases is higher than their axiomatization coverage. Axiomatization coverage can be as low as $1 \%$ for some tests, while code coverage is never less than $25 \%$. That is, there is a certain amount of "core code" exercised by all tests, while there are no "core axioms" used by all tests (this may, of course, be different for other verification systems).

Also, the coverage for other elements of the prelude besides axioms, e.g., type declarations, turned out to be much higher than the axiomatization coverage. It was $81 \%$ for the first experiment and $72 \%$ for the second experiment. It decreased as the new version of the prelude contains declarations related to informationflow analysis, for which no tests have been added to the test suite (yet).

Errors Found in VCC The main goal of our case study was not to find bugs in VCC but to evaluate the quality of the tests. And it was to be expected that no errors could be detected using VCC's own test suite as those tests, of course, had already been used by the VCC developers for testing.

Using tests from other sources (which had a rather low coverage), we found (only) one completeness failure and no soundness failure. The completeness bug in the axiomatization, which has been fixed in the current version of VCC, related to the ownership model. In some situations it was not possible to prove that some part of the state had not changed after an assignment to a memory location outside that part of the state.

\subsection{Testing the Calculus Rules of $\mathrm{KeY}$}

The KeY System As second target for our case studies we have chosen the $\mathrm{KeY}]^{5}$ tool [5], a verification system for sequential Java Card programs. Similar to VCC, programs can be specified using annotations in the source code. In KeY, the Java Modeling Language (JML) is used to specify properties about Java programs with the common specification constructs like pre- and postconditions for methods and object invariants. Like in other deductive verification tools, the verification task is modularized by proving one Java method at a time.

\footnotetext{
${ }^{5}$ See http://www.key-project.org
} 
In the following, we will briefly describe the workflow of the KeY system - in our case, we assume the user has chosen one method to be verified against a single pre-/postcondition pair. First, the relevant parts of the Java program, together with its JML annotations are translated to a sequent in Java Dynamic Logic, a multimodal predicate logic. Validity of this sequent implies that the program is correct w.r.t. its specification. Proving the validity is done using automatic proof strategies within $\mathrm{KeY}$ which apply sequent calculus rules implemented as so-called taclets.

The set of taclets provided with KeY plays a similar role as the prelude in case of VCC, as it captures the semantics of Java, built-in abstract data types like sequences etc.-in comparison to the prelude of VCC, KeY, however, also contains taclets that deal with first order logic formulas, whereas first order reasoning in VCC is handled by the SMT component Z3.

The development version of KeY as of August 2012, contains about 1500 taclets. However, not all of them are available at a time when performing a proof, as some of the taclets exist in several versions, depending on proof options chosen (e.g., handling integer arithmetic depends on whether integer overflows are to be checked or not).

Automatic proof search is combined with interactive steps of the user, in case a proof is not found automatically. For our purposes, the interactive part of KeY is irrelevant, as we restrict test cases to those that can be proven automaticallyotherwise, finding the a minimal set of taclets needed to prove a program correct is infeasible.

Results of a verification attempt in KeY are also similar to those in VCC: either the generated Java Card DL formula is valid and KeY is able to prove it; or the generated formula is not valid and the proof cannot be closed; or KeY runs out of resources.

Axiomatization Coverage Results Using a modified version of our testing framework, we automatically executed the test cases contained in KeY's test suite, as well as parts of a custom Java compiler test suite and measured the taclet coverage.

The procedure used here is similar to reducing VCC's axiomatization. However, the set of taclets to start from is directly taken from the explicit proof object that KeY maintains. The taclet applications recorded for the proof normally contain a lot more taclets than are actually relevant for finding the proof. Thus, we reduce the set of taclets used in the proof construction one by one in a second step in the same manner as for VCC.

Third Experiment. In this experiment we used the development version of the $\mathrm{KeY}$ too ${ }^{6}$ as of August 16, 2012. As part of the KeY source distribution, a test suite is provided containing 335 test cases of which 327 are completeness and 8 are soundness tests. The complexity of the proof obligations ranges from simple arithmetic problems to small Java programs testing single features of Java, up to

\footnotetext{
${ }^{6}$ Available at http://i12www.ira.uka.de/ bubel/nightly/
} 


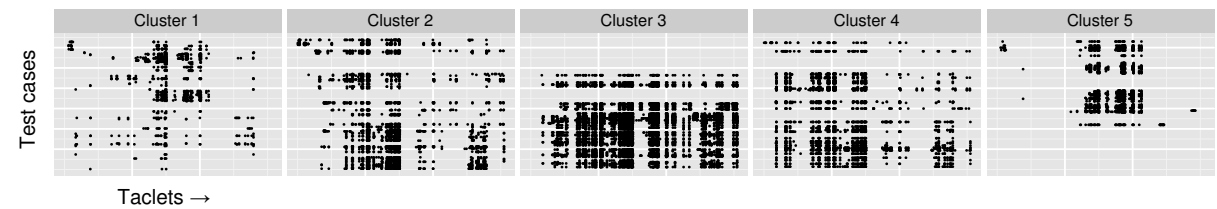

Fig. 1: Groups of similar KeY test cases in terms of taclet coverage. Each point in the diagram indicates a covered taclet by a test case. Clusters computed by hierarchical clustering using Ward's method and Jaccard distance as measure for dissimilarity between taclet coverage of two test cases. $x$-axis sorted by taclet group, $y$-axis sorted by directory a test case is contained in.

more complex programs and properties taken from recent software verification competitions.

From the 327 completeness tests of the KeY test suite, we computed the taclet coverage of 319 test cases testing verification of functional properties - another eight test cases are concerned with the verification of information flow properties and were omitted due to resource constraints. The test runs were distributed on multiple computers using Amazon's Elastic Compute Cloud service, taking approx. 71 EC2 Compute Unit 7 hours to complete in total.

The overall taclet coverage we computed for the 319 completeness tests was $41 \%$ (with 629 out of 1527 taclets covered). Figure 2a shows a histogram of the number of test cases each taclet is covered by. The overall coverage seems to be comparable to the coverage results gained from the VCC test suite. However, in contrast to $\mathrm{VCC}, \mathrm{KeY}$ also performs first order reasoning with the help of taclets instead of using an SMT solver - for better comparison with VCC, all such taclets would have to be excluded from coverage computation.

In order to perform a more detailed analysis, coverage results were examined by groups of taclets respectively test cases. For grouping taclets, we used the already existing structure given by the organization of taclets in different files in the KeY distribution - similar taclets (e.g., taclets handling Java language features or taclets for propositional logic) are contained together in one file.

Histograms of the number of test cases a taclet is covered by, split by taclet group, already allowed us to compare the quality of the test suite w.r.t. the different groups: not surprisingly, taclets handling propositional logic or Java heap properties are covered quite often (in both groups about $70 \%$ of taclets are covered at least in one test case). On the other end of the spectrum, we were able to locate underrepresented taclet groups, e.g., relevant for Java assertions or the bigint primitive type of JML (with coverage of each group below 10\%). This coarse classification already allows to focus the effort of writing new test cases on constructing specific tests for seldomly covered taclet groups.

In order to group similar test cases together to identify commonalities, we used the $\mathrm{R}$ environment for statistical computing [16] to cluster test cases. The result of this clustering is shown in Fig. 1.

\footnotetext{
7 According to Amazon's EC2 documentation, "One EC2 Compute Unit provides the equivalent CPU capacity of a 1.0-1.2 GHz 2007 Opteron or 2007 Xeon processor"
} 


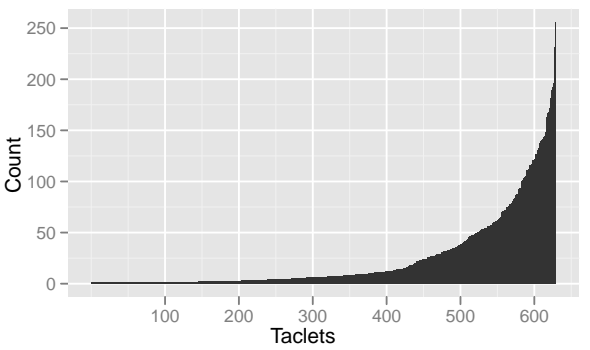

(a) Taclet coverage counts. $y$-axis: number of test cases a taclet is covered by. On the $x$-axis: taclets at least covered by one test case, sorted by $y$ values.

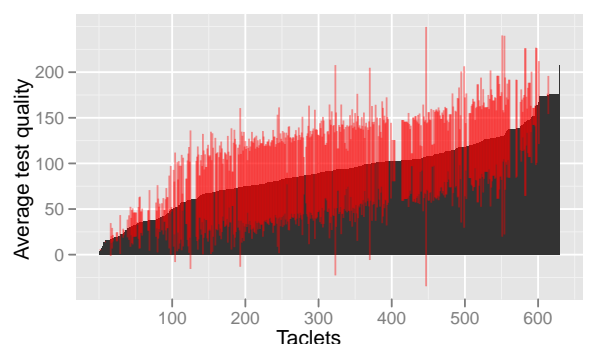

(b) Average test case selectivity by taclet. In black: average selectivity (cf. Sec. 6.2 of all test cases covering a taclet. Deviation of this value from the average is shown in red.

Two of these test case clusters are notably representative for different types of test cases: while the tests in cluster five mostly encompass a small set of related taclets, tests in cluster three span almost the entire taclet base of KeY. Indeed, test cases belonging to cluster five have been written as specific tests for single features of $\mathrm{KeY}$ - in this case, integer arithmetic and handling strings (which need similar taclets because the functions retrieving characters from a string or getting substrings use integers; the small group of taclets in the upper left corner of cluster five are the taclets handling strings). Test cases corresponding to cluster three are mostly taken from verification competitions dealing with data structures on the heap like linked lists.

We believe that a good test suite for a verification system needs both of these types of test cases for each taclet. The need for broader test cases, covering several combinations of taclets, is supported by studies (e.g. [14]) which show that software failures in a variety of domains are often caused by combinations of several conditions. Specialized test cases, in comparison, might simplify testing different aspects of one taclet by being able to better control the context a taclet will presumably be applied in the proof. As a measure for this, we define the selectivity of a test case as the number of taclet covered by the test.

The current state of the $\mathrm{KeY}$ test suite w.r.t. this selectivity criterion is shown in Fig. 2b. For each taclet, the average selectivity of all test cases covering this taclet is shown, together with the deviation from the average. The leftmost taclets in this diagram are good candidates for which additional test cases might be needed, as they are only covered by specialized test cases. Also taclets with a high selectivity average of the corresponding test cases but low deviation indicate need for improvements, as only broad test cases cover the taclets.

Fourth Experiment. The last experiment we conducted used parts of the test suite of the Java2Jinja ${ }^{8}$ compiler [15] in order to increase taclet coverage compared to KeY's own test suite.

\footnotetext{
${ }^{8}$ Available at http://pp.info.uni-karlsruhe.de/projects/quis-custodiet/ Java2Jinja/
} 
The part of Java2Jinja's test suite we considered here is hand-written and consists mostly of small Java programs testing few Java features at a time, often dealing with corner cases of the Java semantics. All tests are run by providing concrete, fixed input parameters to the test's main method. The result of the execution of a test is an output to the console - the expected outcome of the test case is given as annotation in the test file. This annotation can easily be converted into a postcondition suitable to be proven with $\mathrm{KeY}$.

From a total of 43 annotated test cases, 21 were applicable to $\mathrm{KeY}$-most of the other tests included features not yet supported by KeY (e.g., Java generics). Of the 21 suitable tests, 12 were directly provable (two thereof using user interaction), the rest of the proofs exceeded allocated time or memory resources.

One result of the test runs was that the Java2Jinja test suite covered 195 of 1527 taclets, corresponding to $13 \%$ taclet coverage. Even this small set of additional test cases covered nine taclets that were not covered by KeY's own test suite.

Errors Found in KeY As already argued in Sect. 6.1, we could not expect to find soundness bugs using KeY's own test suite. Therefore, we performed the last experiment using Java2Jinja's test cases - resulting in two bugs found in the rule base of $\mathrm{KeY}$.

The first bug we discovered is related to implicit conversion of integer literals to strings in Java: in case a negative integer literal is converted to its string representation, the minus sign is placed at the last instead of first position in the resulting string. The Java2Jinja test case that revealed this bug has not been written to test exactly this conversion feature but rather the correct handling of precedences in integer arithmetic. To write an additional test case for this exact feature that increases axiomatization coverage is easy, as exactly one taclet is involved in the bug and the condition under which this taclet is applicable is clearly visible to the user.

The second bug found in the taclets of $\mathrm{KeY}$ deals with the creation of inner member classes, using a qualified class instance creation expression. However, the corresponding taclet in $\mathrm{KeY}$ that symbolically executes this instance creation expression does not check whether the qualifying expression is null and always creates an object of the inner class without throwing the required NullPointerException (see the Java language specification, Sect. 15.9.4).

While both of these bugs in the taclets allow the user to prove properties about certain programs that do not hold, they do not lead to unsoundness in the general case: both bugs are only triggered if the Java program contains the corresponding features (i.e., qualified class instance creation or conversion of negative integer literals to string). In addition, the bugs only influence the correctness of the proof if the property to be verified relies those exact features. In both cases, the KeY test suite did not cover the relevant taclets at all, and the increase in the axiomatization coverage by the two relevant Java2Jinja tests indeed allowed to reveal faulty taclets. This shows that axiomatization coverage is a useful metric to get a first hint to parts of the axiomatization that may be target for further inspection and validation measures. 


\section{Related Work}

In principle, instead of or in addition to testing, parts of verification tools (in particular the axiomatization and the calculus) can be formally verified. For example, the Bali project [17, the LOOP project [12], and in the Mobius project [3], all aimed at the development of fully verified verification systems. Calculus rules of the KeY verification system [5] for Java were verified using the Maude tool [1]. Verifying a verification system is useful, but it cannot fully replace testing; this is further discussed in 6 .

Another approach to reveal inconsistencies that, for example, the VCC tool provides is a mode in which the tool tries to derive false from the axiomatization. That is a useful feature complementing testing. But it cannot replace all soundness tests. Not all inconsistencies are found (due to incompleteness) and not every unsoundness leads to inconsistency.

For the theorem provers and SMT solvers that are components of verification systems, there are established problem libraries that can be used as test suites, such as the SMT-LIB library [2]. Alternatively, the results of SMT solvers can be validated using proof checkers. For example, Z3 proofs can be checked using Isabelle [7]. Another example is the Formally Verified Proof Checker that was implemented in ML formally verified using HOL88 [18].

An interesting application of conformance testing is the official validation test suite for FIPS C (a dialect of C) 13. In order to determine the coverage of the language standard by a test suite, the standard was implemented in a model implementation. Code within this model implementation was then mapped back to the standard, allowing the authors to show that all of the requirements in the standard were implemented. Moreover, code coverage w.r.t. the model implementation thus relates to coverage of the language standard.

\section{Conclusions}

In this paper, we introduced axiomatization coverage as a new coverage criterion in testing verification systems. Our case studies show that this is a useful and important criterion and should be used as a first measure to judge and improve the quality of test suites.

For the future, we plan to conduct additional case studies to further evaluate our coverage criteria and, in particular, to investigate which kind of tests uncover what kind and what number of errors. A first step in this direction has been made using parts of the Java2Jinja test suite, which revealed two bugs in KeY's rule base. Additional test suites not written by tool developers themselves are required though to get useful statistics about bug occurrences.

We plan to investigate the reasons why some axioms are not covered, amongst others, using the help of developers of the verification systems. Afterwards, an experiment is planned to be conducted where we systematically write specific test cases aimed to increase the axiomatization coverage for relevant axioms of the existing test suites. If our assumption that axiomatization coverage is a useful measure is right, we should be able to find further bugs with these tests. 
Further, we plan to use combinatorial testing, where combinations of language features and axioms are used in test cases, as well as more fine-grained axiomatization coverage criteria, based on coverage of axioms as a whole.

Acknowledgements We thank Andreas Lochbihler, Jonas Thedering and Antonio Zea for providing the Java2Jinja test cases.

\section{References}

1. W. Ahrendt, A. Roth, and R. Sasse. Automatic validation of transformation rules for Java verification against a rewriting semantics. In $L P A R$ '05, volume 3835, pages 412-426. Springer, 2005.

2. C. Barrett, S. Ranise, A. Stump, and C. Tinelli. The satisfiability modulo theories library (SMT-LIB). At http://www.smt-lib.org/.

3. G. Barthe, L. Beringer, P. Crégut, B. Grégoire, M. Hofmann, P. Müller, E. Poll, G. Puebla, I. Stark, and E. Vétillard. MOBIUS: Mobility, Ubiquity, Security, volume 4661 of $L N C S$. Springer, 2006.

4. B. Beckert, T. Bormer, and V. Klebanov. Improving the usability of specification languages and methods for annotation-based verification. In B. Aichernig, F. S. de Boer, and M. Bonsangue, editors, FMCO 2010. State-of-the-Art Survey, volume 6957 of LNCS. Springer, 2011.

5. B. Beckert, R. Hähnle, and P. H. Schmitt, editors. Verification of Object-Oriented Software: The KeY Approach, volume 4334 of LNCS. Springer-Verlag, 2007.

6. B. Beckert and V. Klebanov. Must program verification systems and calculi be verified? In 3rd International Verification Workshop (VERIFY), Workshop at Federated Logic Conferences (FLoC), pages 34-41, 2006.

7. S. Böhme. Proof reconstruction for $\mathrm{Z3}$ in Isabelle/HOL. In 7th International Workshop on Satisfiability Modulo Theories (SMT'09), 2009.

8. E. Cohen, M. Dahlweid, M. Hillebrand, D. Leinenbach, M. Moskal, T. Santen, W. Schulte, and S. Tobies. VCC: A practical system for verifying concurrent C. In TPHOLs'09, volume 5674 of LNCS, pages 23-42. Springer, 2009.

9. S. A. Cook. Soundness and completeness of an axiom system for program verification. SIAM Journal of Computing, 7(1):70-90, 1978.

10. L. de Moura and N. Bjørner. Z3: An efficient SMT solver. In TACAS'08, LNCS 4963, pages 337-340. Springer, 2008.

11. J.-C. Filliâtre and C. Marché. Multi-prover verification of C programs. In Formal Methods and Software Engineering, LNCS 3308, pages 15-29. Springer, 2004.

12. B. Jacobs and E. Poll. Java program verification at Nijmegen: Developments and perspective. LNCS, 3233:134-153, 2004.

13. D. Jones. Who guards the guardians?, Feb. 1997.

14. D. R. Kuhn, D. R. Wallace, and A. M. Gallo. Software fault interactions and implications for software testing. IEEE Transactions on Software Engineering, 30(6):418-421, 2004.

15. A. Lochbihler. A Machine-Checked, Type-Safe Model of Java Concurrency. PhD thesis, Karlsruhe Institute of Technology, to appear.

16. $\mathrm{R}$ Core Team. R: A Language and Environment for Statistical Computing. $\mathrm{R}$ Foundation for Statistical Computing, Vienna, Austria, 2012.

17. D. von Oheimb. Hoare logic for Java in Isabelle/HOL. Concurrency and Computation Practice and Experience, 13(13):1173-1214, 2001.

18. J. von Wright. The formal verification of a proof checker. SRI internal report, 1994. 